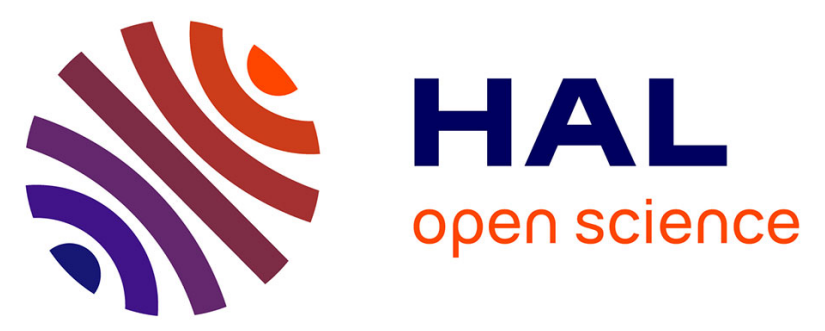

\title{
Dynamic Short-Range Sensing Approach using MIMO Radar for Brain Activities Monitoring
}

Mohammad Ojaroudi, Stéphane Bila

\section{To cite this version:}

Mohammad Ojaroudi, Stéphane Bila. Dynamic Short-Range Sensing Approach using MIMO Radar for Brain Activities Monitoring. 2020 14th European Conference on Antennas and Propagation (EuCAP), Mar 2020, Copenhagen, Denmark. pp.1-5, 10.23919/EuCAP48036.2020.9135454 . hal-03047710

\section{HAL Id: hal-03047710 \\ https://hal-unilim.archives-ouvertes.fr/hal-03047710}

Submitted on 8 Dec 2020

HAL is a multi-disciplinary open access archive for the deposit and dissemination of scientific research documents, whether they are published or not. The documents may come from teaching and research institutions in France or abroad, or from public or private research centers.
L'archive ouverte pluridisciplinaire HAL, est destinée au dépôt et à la diffusion de documents scientifiques de niveau recherche, publiés ou non, émanant des établissements d'enseignement et de recherche français ou étrangers, des laboratoires publics ou privés. 


\title{
Dynamic Short-Range Sensing Approach using MIMO Radar for Brain Activities Monitoring
}

\author{
Mohammad Ojaroudi ${ }^{1}$, and Stéphane Bila ${ }^{1}$ \\ ${ }^{1}$ XLIM, UMR no7252, University of Limoges, 123 Av. Albert Thomas, 87060 Limoges, France \\ e-mail: mohammad.ojaroudi@xlim.fr
}

\begin{abstract}
This paper presents a new concept of functional microwave imaging using m-sequence multiple-input multipleoutput (MIMO) radar as a non-ionizing application of functional brain imaging. The underlying hypothesis is that, if we can detect local changes in blood volume inside the brain precisely enough, we can infer which parts of the brain are activated when performing various tasks. In this point of view, the main challenge in terms of MIMO radar framework is multi-target localization based on time of arrival (TOA) results. For this purpose, we present a multi-lateral localization approach in collocated MIMO-radar to detect single target inside brain medium. A system concept is introduced, and results from simulations using a simplified physical model are presented. To validate this, we focus on the waveform diversity and signaling strategies options for short range sensing. Simulated results validate the effectiveness of the proposed methods for precisely calculating the time-dependent location of target.
\end{abstract}

Index Terms - colocated multi-input multi-outptut (MIMO) framework, functional microwave imaging system, time of araival (TOA), multi-lateral localization, m-sequence pseudo noise radar.

\section{INTRODUCTION}

Among neuro imaging techniques, functional magnetic resonance imaging (fMRI) is currently the most widely used as it can provide excellent detailed anatomical and physiological information related to pathologies such as brain tumors, Alzheimer's disease, epilepsy and etc. [1-2]. Due to their mechanical complexity and size, magnetic resonance imaging is not practical for long term continuous monitoring. In addition, metallic objects are prohibited, thereby excluding patients with a surgical prosthesis or pace maker [3-5]. In other words, fMRI scanners are excessively large and expensive. Although medical technology is progressing fast and researchers continue their efforts on finding new methods to study the brain activities, the need for a non-invasive, lowcost and portable system for long term monitoring is as present as ever before.

Multi-input multi output (MIMO) based microwave imaging system has great potential to provide the means of portable, non-invasive, noncontact long-term continuous monitoring of cancerous tissues, brain stroke tomography, and even functional neuro imaging which are not possible using other existing techniques [6]. The separation of multiple incident signals, can greatly increase flexibiliry and capacity for systems performance [7]. For the brain monitoring applications, research is needed on multi-sensor detection in the presence of interfrence and heavy clutter, the exploitation of path diversity in the biological channel by techniques like space-time coding for hostile environments, and modulation identification. A challenging problem and key issue in MIMO imaging radar is resolution improvement. The waveform diversity paradigm in the MIMO imaging radar area involves the use of appropriate incident pulse shape based on variation of a practical environment [8-9]. Using orthogonal msequence signals can be consider as a logical extension from channel estimation to channel exploitation. In this context the characteristics of the physical environment are estimated and the modulation scheme or the transmitting waveform are modified to maximize the likelihood of reception at the other end of the link [10].

In this paper, we explore the advantages of orthogonal waveform diversity in collocated MIMO radar for target detection inside brain medium. The advantage conferred by using m-sequence orthogonal signals waveform diversity in the MIMO framework is that the resolution and accuracy of target localizing for time domain reflected is improved. Data are acquired by illuminating the brain multi-layer structure with $\mathrm{m}$-sequence orthogonal signals and then recording the backscattered fields using MIMO array. In this context, this paper aims to provide a multi-lateral localization in order to focus on multipath reflected signals that will be used in functional microwave brain imaging systems. Simulated results validate that the MIMO based framework could be a good candidate for brain activities monitoring.

\section{M-SEQUENCE BASED MIMO RADAR FRAMEWORK FOR BRAIN ACTIVITIES MONITORING}

The underlying hypothesis of functional microwave imaging for brain activities monitoring is that, by detecting local changes in blood volume inside the brain precisely enough, we can indicate which parts of the brain's regions are activated when performing various tasks such as moving various muscles, making decisions, experiencing emotions and others [11]. Fig. 1 shows brain blood vasculature as a function of blood flow. Neural activity increases the oxygen and glucose consumption and they generates Hemodynamic response. It cause dilation of blood vessels around 20-50\% and increasing cerebral blood volume $<6 \%$ [12]. 

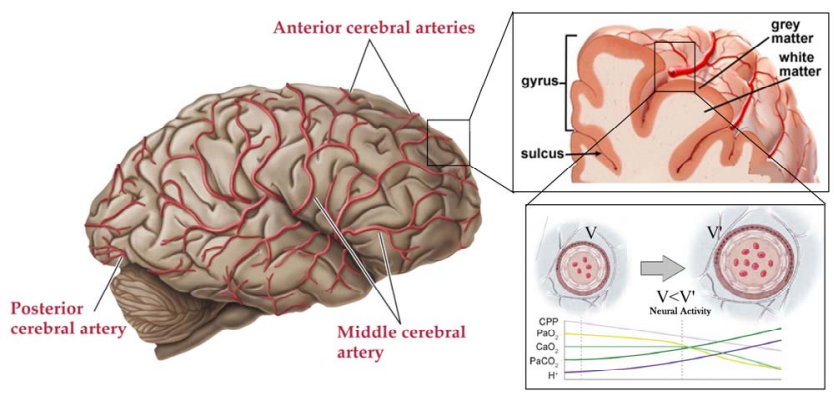

Fig. 1. Brain blood vasculature as a function of blood flow.

The proposed envisioned functional microwave imaging system's architecture is shown in Fig. 2, which includes the flow of the m-sequence MIMO processing in the envisioned system to detect brain activities. This framework includes switching network blocks of $\mathrm{m}$-sequence signal generator and antenna array. In order to collect 3D scattered samples from multi-layer biological tissues, a 3D switched antenna array configurations using particular multiplexed sequences can be used to cover the human head hemispherical surface. When the brain is completely scanned, the computer generates $3 \mathrm{D}$ image from the measured values through confocal image reconstruction method. Regarding time-domain scanning aims, the high levels of scatter and corresponding multipath interferences in near field shall be the main concern. In post processing section, image-processing techniques can be performed to analyses the observed variations of blood volume. Through every scan, the generated model is upgraded and modified as necessary.

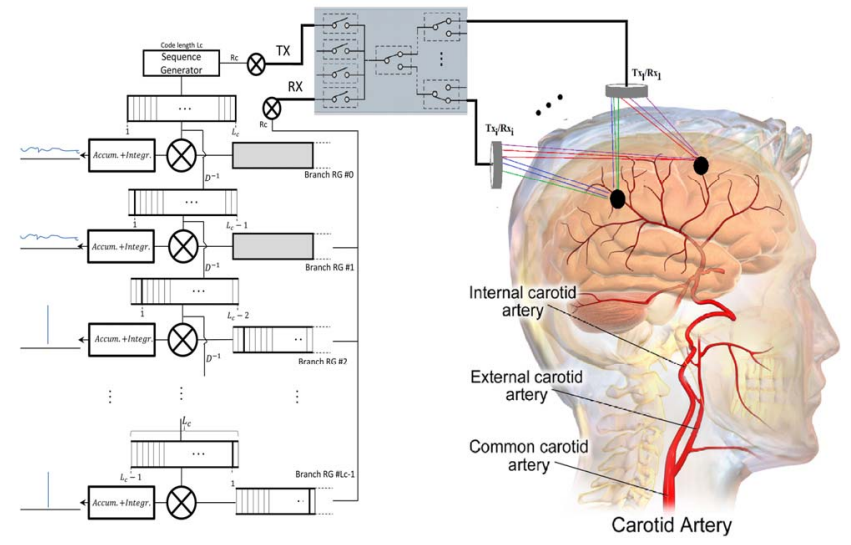

Fig. 2. The flow of the m-sequence MIMO processing in the envisioned framework.

\section{SYSTEM MODEL AND PROBLEM FORMULATION}

In this section, the proposed system model and problem formulation for the time of arrival (TOA) based multipletarget localization algorithm is presented. TOA measurement provides information about the distance between the reference and the target by estimating the time of flight of a signal that travels from one to the other. In the MIMO framework, where the target just reflects the transmitted signals from reference position. In this case, TOA expresses the signal propagation time from the transmitter towards a target and reflected from the target towards the receiver $(\mathrm{Rx})$.
This defines an ellipse with focal in transmitting node and receiving node and the semi major axis equal to the half of TOA estimated distance [13].

\section{A. Time of Arrival based MIMO system for Target Detection}

For the $m^{\text {th }}$ of all $M$ transmitter antenna the waveforms can be written as $p_{\mathrm{m}}[k], m=1,2, \ldots, M$. In the case of single target scenario, the received signal from the $n^{\text {th }}$ receiver antenna can be written as:

$$
r_{n}[k]=\sum_{m=1}^{M} p_{m}\left[k-\tau_{n m} / T_{S}\right]+W_{n}[k]
$$

Where $\tau_{n m}$ indicates the ToA information for the waveform that propagates through the brain medium related to the $m^{\text {th }}$ transmitter antenna and the $n^{\text {th }}$ receiver antenna. $W_{n}[k]$ can be considered as the noise signal of the associated receiver antenna. $T_{s}$ is the sampling period. In order to obtain the arrival time $\tau_{n m}$ of the transmitted signal from the $m^{\text {th }}$ transmitter antenna to the $n^{\text {th }}$ receiver antenna, sampled signal $r_{n}[k]$ will pass through a receiving matched filter $g_{m}[k]$ as follows:

$$
\gamma_{n m}[k]=r_{n}[k] * g_{m}[k]=\left(\sum_{m^{\prime}=1}^{M} p_{m^{\prime}}\left[k-\tau_{n m^{\prime}} / T_{S}\right]\right) *
$$

Where $*$ denotes convolution operation and $\mathrm{g}_{\mathrm{m}}[\mathrm{k}]$ is the impulse response of the $m^{\text {th }}$ receiving matched filter. The correlation between the transmitted signal $p_{m}\left[k-\tau_{n m} / T_{S}\right]$ and the receiving matched filter $g_{m}[k]$ will produce a correlation peak. The position of the peak indicates the ToA of the reflected signals. During a single excitation, several orthogonal signals will be transmitted [14]. By combining the returns from all these waveforms echoed by the given targets during one excitation, the SNR of the integrated waveforms can be increased. The coherent integration can be calculated as follows:

$$
\gamma_{n m}^{\prime}[k]=\left\|\sum_{k_{0}}^{K} \gamma_{n m}\left[k+k_{0} \times L\right]\right\|^{2}
$$

Where \| \|indicates the Euclidean norm. $L$ is the length of each copy of the transmitted waveforms. Based on coherent integration, the power of the radar signal is unchanged since all transmitting signals are the same. Hence, coherent integration would nominally provide $10 \log 10 \mathrm{np} \mathrm{dB}$ of SNR improvement. ToA, $\gamma^{\prime}{ }_{n m}$ for the waveform from the $m^{\text {th }}$ transmitter antenna to the $n^{\text {th }}$ receiver antenna in the single target scenario, can be estimated by searching the maximum value of correlation result as follows:

$$
\tilde{r}_{n m}=\arg \max _{k}\left\|\gamma_{n m}^{\prime}[k]\right\|^{2}
$$

The sign ( $\left.{ }^{\sim}\right)$ referred to an estimated value.

\section{B. Multiple-Target Localization Algorithm}

In the situation with multiple targets or with multi-layer structure like brain, ToAs cannot be estimated by simply searching the maximum value of $\gamma_{n m}^{\prime}[k]$. There will be multiple correlation peaks in one $\gamma^{\prime}{ }_{n m}[k]$ due to the multiple targets. A threshold can be used for detection in this case. All of the positions where the correlation output is higher than the threshold should be included into the ToA list. In this case, for an $N \times M$ antenna setup, there are $N \times M$ entries in measured ToA matrix. Each entry $\tilde{r}_{n m}$ is the ToA in the 
associated antenna pair. From the ToA information $\tilde{r}_{n m}$ in one channel, the position of all detectable target locations of the associated channel can be captured. In bistatic radar, the locus is an ellipse whose focal points are the locations of the pair of antennas related to the specific ToA.

The possible target position $(\bar{x}, \bar{y})_{n m}$ is on the ellipse and it should satisfy the following nonlinear equation:

$$
\tilde{r}_{n m}=\frac{1}{v}\left\|\left(x_{t m}, y_{t m}\right)-(\bar{x}, \bar{y})_{n m}\right\|+\frac{1}{v}\left\|\left(x_{r n}, y_{r n}\right)-(\bar{x}, \bar{y})_{n m}\right\|
$$

For $n=1,2 \ldots N$ and $m=1,2, \ldots M$, the sign $v$ represents the speed of wave in the brain medium and $\tilde{r}_{n m}$ represents the ToA. The position of the transmitter antenna is expressed as $\left(x_{t m}, y_{t m}\right)$ while the receiver antenna position is $\left(x_{r n}, y_{r n}\right)$. The crossing positions $(\hat{x}, \hat{y})$ of the ellipses designate the approximated target position. It can be obtained by solving the optimization problem as follow.

$$
\begin{aligned}
& \underset{(\hat{x}, \hat{y})}{\operatorname{argmin}} \sum_{n=1}^{N} \sum_{m=1}^{M}\left(\left\|(\hat{x}, \hat{y})-(\bar{x}, \bar{y})_{n m}\right\|\right) \\
& \text { Subject to } \quad \text { to } \quad \tilde{r}_{n m}=\frac{1}{v}\left\|\left(x_{t m}, y_{t m}\right)-(\bar{x}, \bar{y})_{n m}\right\|+ \\
& \frac{1}{v}\left\|\left(x_{r n}, y_{r n}\right)-(\bar{x}, \bar{y})_{n m}\right\| ; n=1,2, \ldots, N ; m=1,2, \ldots, M .
\end{aligned}
$$

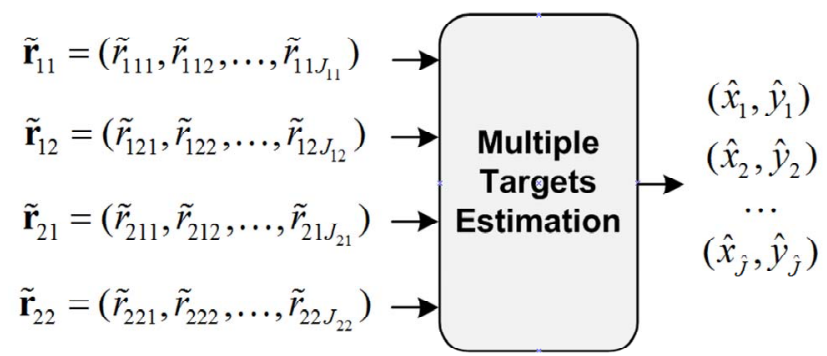

Fig. 3. Data flow of the localization algorithm for multiple targets [14].

This nonlinear optimization problem can be solved by the Levenberg-Marquardt algorithm (LMA) [15]. The LMA supplies a numerical solution to the issue of minimizing a non-linear function. Data flow of the localization algorithm for multiple targets is shown in Fig. 3. LMA is the least squares curve iterative method. In the first sounding, the initial value is set to the central position of the experiment field. In the following illumination, the initial value is set to the localization result of the last illumination. In a multiple target condition, each $\tilde{r}_{n m}$ contains several ToAs related to corresponding targets.

$$
\tilde{r}_{n m}=\left(\tilde{r}_{n m 1}, \tilde{r}_{n m 2}, \ldots, \tilde{r}_{n m q_{n m}}\right)
$$

Where $q_{n m}$ is the number of ToAs included in $\tilde{r}_{n m}$.

\section{PERformance Evaluation of MUlti-Lateral RELATIONSHIP IN COLOCATED-MIMO SCENARIO}

This section presents the simulated scenario of the proposed m-sequence MIMO radar system for target localization. In the simulated scenario, as shown in Fig. 4, a target is surrounded by three transceivers. The computing processor has been implemented in MATLAB running computer.

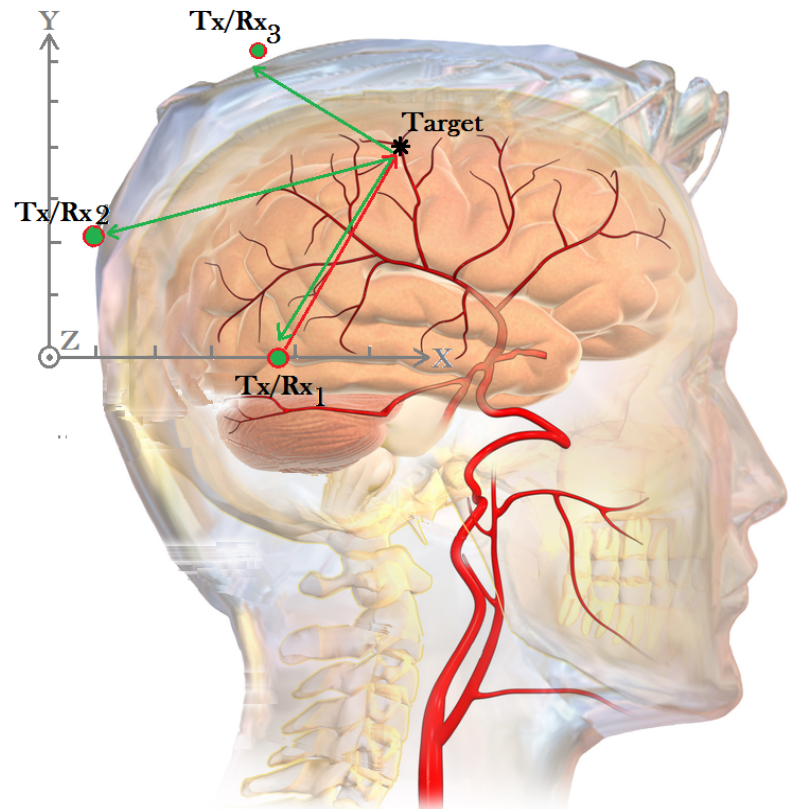

Fig. 4. The positions of target and antennas for MIMO imaging (grid's unit: $\mathrm{cm})$.

In this simplified simulation study, we consider three antennas and single target to demonstrate the effectiveness of the proposed framework to develop the vascular target location estimation algorithm. The actual position of the target and antennas are marked in Fig. 4. The elements of the 3-antennas collocated MIMO array are close to the brain that sit $2 \mathrm{~cm}$ away from the skin layer. A single spherical target with radius of $0.5 \mathrm{~cm}$ is placed at the brain surface that indicates the dilation of blood vessels. First, three Pseudo noise (PN) m-sequence that are highly orthogonal to each other has been selected to be the sounding pulses. For this purpose, the brain multi-layer is generated by MATLAB and the orthogonal signals are added as an excitation. Three PN $\mathrm{m}$-sequences signals and their normalized autocorrelation and cross-correlation functions between the waveforms and the receiving filters are shown in Fig. 5 and Fig. 6, respectively.
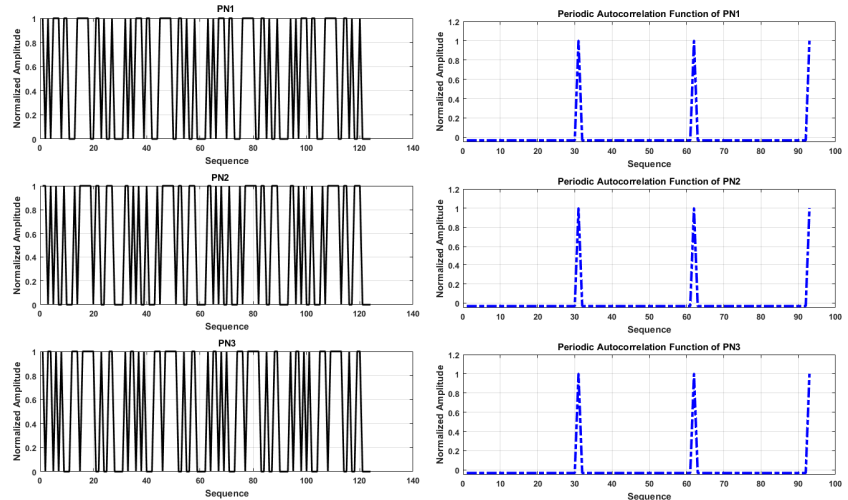

Fig. 5. Three PN m-sequences signals and their normalized autocorrelation, which are highly orthogonal to each other. 


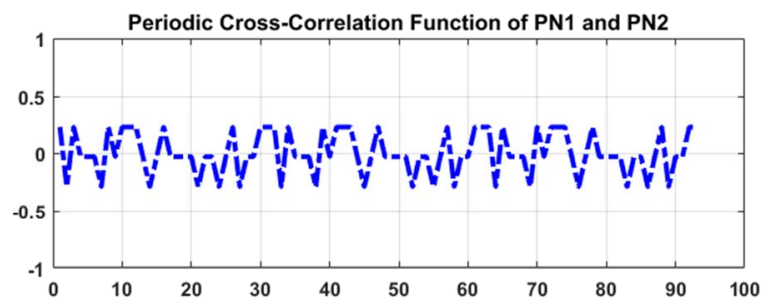

(a)

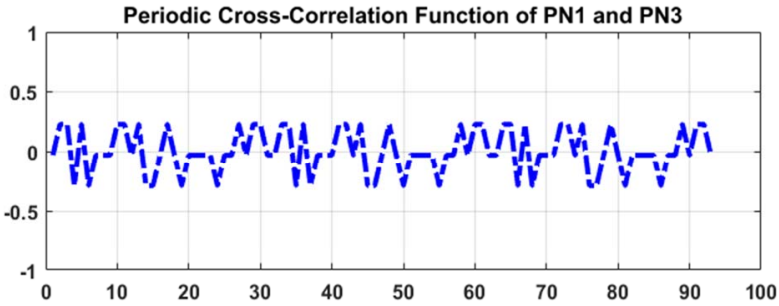

(b)

Fig. 6. Two samples of the normalized cross-correlation functions between the, (a) $\mathrm{PN}_{1}$ and $\mathrm{PN}_{2}$, and (b) $\mathrm{PN}_{1}$ and $\mathrm{PN}_{3}$.

In addition, Fig. 7 introduces the multi-layer environment schematic of the proposed multi-reflection based short range sensing approach of MIMO target detection. In order to simplicity in simulation we have used a configuration of a hemispherical biological tissue phantom (radius: $12 \mathrm{~cm}$ ) with a spherical target (radius: $0.5 \mathrm{~cm}$ ) in the CSF layer of the head model.

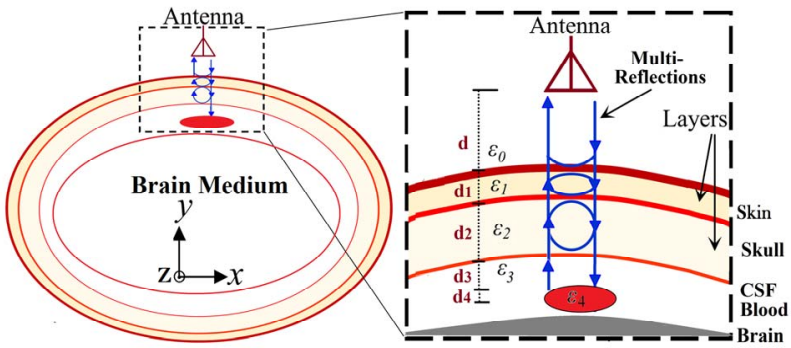

Fig. 7. Brain medium with vascular target as a multilayer medium with multi reflection characteristics as reference geometry for MIMO propagation model used in the analysis.

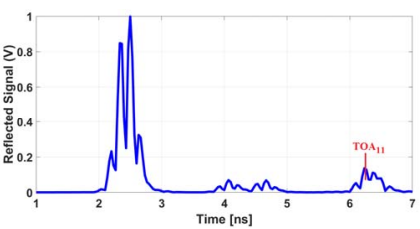

(a)

(c)

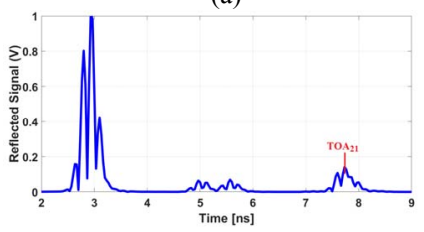

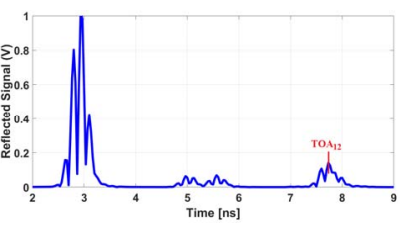

(b)

(d)

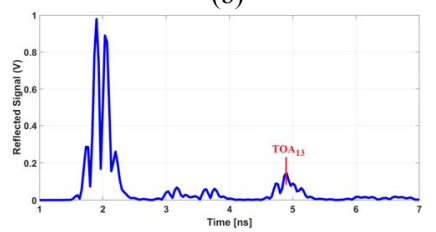

Fig. 8. Four samples of the reflected signals $\left(\mathrm{r}_{\mathrm{ii}}\right)$ when a single target is in the scenario for computing the TOA's positions, (a) $r_{11}$, (b) $r_{12}$, (c) $r_{21}$, and (d) $\mathrm{r}_{13}$.

The choice of the central frequency and the bandwidth of the m-sequence radar are the key factors in the imaging or tracking system design. Although the higher frequencies are needed for better resolution and detailed echo to determine small size objects, the lower frequencies are preferred to detect something buried too deep because of the dramatically increased attenuation of the medium with increasing frequency. Thus, the m-sequence pulses covering $1-5 \mathrm{GHz}$ is used in order to offer a balance between the two contradictory parameters needed for better spatial resolution and better penetration depth [16]. This frequency range sets a theoretical spatial resolution as an inter-sample distance of less than 2 $\mathrm{mm}$, which is less than the conventional fMRI systems spatial resolution (3-5 $\mathrm{mm})$.

The reflected signals are obtained when there is a target in the simulated multi-layer brain scenario as shown in Fig. 8. According to the simulation scenario, the last maximum point of each output signals represents corresponding TOA of the target. In this study in order to simplify formulation, a monostatic scenario of the proposed MIMO framework is used. For the MIMO approach, the processing algorithms discussed earlier operate as expected, since the monostatic approach is one particular case of bistatic. Because of MIMO nature, these algorithms can apply to arbitrarily placed transmitters and receivers, thus giving it a low setup cost. To implement the proposed MIMO as a monostatic radar, the transmitter and receiver can be set to the same coordinates in the simulation. This can be beneficial by requiring fewer antennas as transmitters and receivers.

By using the ellipse-positioning algorithm, the estimated position of target can be obtained. For this purpose, simulated results of TOA-based ellipse-positioning algorithm using monostatic scenario radar network in MATLAB are shown in Fig. 9. The TOA algorithm assumes that single target are presented at several antenna locations [17]. This cluster of antennas is referred to the initialization window. Associated with each response is an estimate of the received target's TOA. As shown in Fig. 9, the actual position of the target and antennas are marked as well as the estimated target position. It can prove that the system works as expected with millimetres range error.

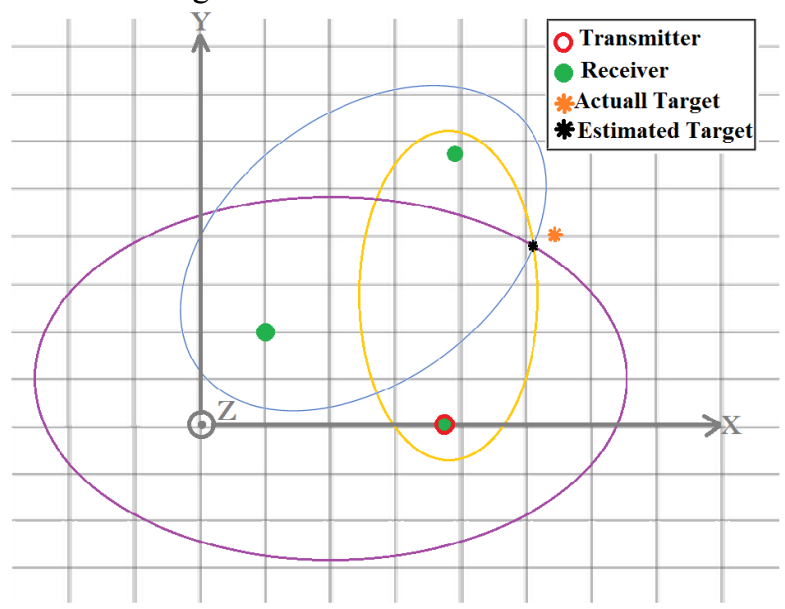

Fig. 9. Localization result for the collocated-MIMO radar system based on ellipse multilateral approach. 


\section{CONCLUSION}

This paper explores and assesses a novel target localization algorithm on envisioned MIMO radar framework for brain activities monitoring application. The localization of vascular target in the surface of the brain cortex is investigated based on orthogonal m-sequence taime-domain transmitted signal combined with MIMO framework as a dynamic short range sensing. In order to show the effects of waveform diversity in MIMO radar we categorized auto, cross-correlation functions of the transmitted and received signal. In addition, we proposed time of arrival (TOA) based ellipse-positioning technique for improving resolution of the received signal. The advantage conferred by "MIMO radar framework" is that based on coherent integration, high signal to noise ratio (SNR) and high-strength is available at reflected signal than with conventional transceivers. Subsequently a relatively higher resolution in identifying target position from the reflected signal is achieved. Results show the proposed approach can offer significant benefits for intra-brain target localization, ensuring enhanced resolution, precision in the captured TOAs.

\section{REFERENCES}

[1] J. Olesen, A. Gustavsson, M. Svensson, H. U. Wittchen, B. Jönsson, CDBE2010 Study Group and European Brain Council, The economic cost of brain disorders in Europe. European journal of neurology, 19(1), pp.155-162, 2012.

[2] J. Beutel, L. Harold, and L. Richard. Handbook of medical imaging. Vol. 1. Spie Press, 2000.

[3] K. Doi, Computer-aided diagnosis in medical imaging: historical review, current status and future potential. Computerized medical imaging and graphics, 31(4-5), pp.198-211. 2007.

[4] M. Guardiola, L. Jofre, F. Gedda, S. Capdevila, J. Romeu, and S. Blanch, 3D arrayed microwave tomographic system for medical imaging. In Wireless Information Technology and Systems (ICWITS), 2010 IEEE International Conference on, pp. 1-4, August 2010.

[5] J. R. Dalenberg, H. R. Hoogeveen, and M. M. Lorist, Physiological Measurements: EEG and fMRI. In Methods in Consumer Research, Volume 2 (pp. 253-277). 2018.

[6] M. Ojaroudi, S. Bila and M. Salimi, "A Novel Approach of Brain Tumor Detection using Miniaturized High-Fidelity UWB Slot Antenna Array," 13th European Conference on Antennas and Propagation (EuCAP), Krakow, Poland, pp. 1-5, 2019.

[7] M. Ojaroudi, S. Bila, P. Leveque and P. Carré, "Functional Microwave Imagining System based on Cognitive Scanning for Brain Activities Monitoring: A Feasibility Study," 13th European Conference on Antennas and Propagation (EuCAP), Krakow, Poland, pp. 1-5, 2019.

[8] J. Sachs, S. Ley, T. Just, S. Chamaani, and M. Helbig. "Differential ultra-wideband microwave imaging: Principle application challenges." Sensors 18, no. 7, pp. 21-36, 2018.

[9] M. Aftanas, J. Rovnakova, M. Riskova, D. Kocur, and M. Drutarovsky, "An analysis of 2D target positioning accuracy for Msequence UWB radar system under ideal conditions." 17th International Conference Radioelektronika, pp. 1-6, 2007.

[10] H. Godrich, A. M. Haimovich, and R.S. Blum, "Target localization accuracy gain in MIMO radar-based systems." IEEE Transactions on Information Theory, 56(6), pp.2783-2803, 2010.

[11] T. Lauteslager, N. Nicolaou, T. S. Lande and T. Constandinou, Functional neuroimaging using UWB impulse radar: A feasibility study. In Biomedical Circuits and Systems Conference (BioCAS), 2015 IEEE (pp. 1-4), October 2015.

[12] T. Lauteslager, M. Tømmer, T. S. Lande, and T. Constandinou, "Coherent UWB Radar-on-Chip for In-Body Measurement of Cardiovascular Dynamics." IEEE transactions on biomedical circuits and systems, 2019.

[13] X. Li, "Ultra-wideband MIMO cognitive sensing systems, algorithms, data processing and testbed." $\mathrm{PhD}$ thesis, DOI: 10.13140/RG.2.1.2658.8567, February 2014.

[14] X. Li, Z. Hu, R. C. Qiu, Z. Wu, J. P. Browning, and M. C. Wicks. "Demonstration of cognitive radar for target localization under interference." IEEE Transactions on Aerospace and Electronic Systems 50, no. 4, pp. 2440-2455, 2014.

[15] X. Li, Z. Hu, and R. C. Qiu. "MIMO UWB Radar System with Compressive Sensing." arXiv preprint arXiv:1604.04291, 2016.

[16] J. Goense, Y. Bohraus, and N. K. Logothetis. "fMRI at high spatial resolution: implications for BOLD-models." Frontiers in computational neuroscience 10, 66, 2016.

[17] M. Švecová, D. Kocur, N. Uramová, and J. Fortes. "TOA complementing method for target localization by UWB radar systems." 16th International Radar Symposium (IRS), pp. 949-954, 2015. 